\title{
Ice-formation phenomena for water flow inside a cooled parallel plate channel: an experimental and theoretical investigation of wavy ice layers
}

\author{
B. WEIGAND and H. BEER \\ Instıtut fur Technısche Thermodynamı. Technische Hochschule Darmstadt. Petersenstraße 30. \\ 6100 Darmstadt. F R.G.
}

(Receited 21 Fehruary 1992)

\begin{abstract}
A numerical model is developed for predictıng steady-state ıce formation insıde a cooled twodimensional channel The study takes into account the strong interactions existing between the turbulent flow, the shape of the ice and the heat transfer at the ice-water interface which lead to the formation of wavy ice layers with one wave. The presented analysis is found to be able to predict realistic variations of the ice layer thickness for a wide range of Reynolds numbers and cooling parameters. The numerical results were verified by comparing the predicted ice layers with measurements and generally good agreement was found.
\end{abstract}

\section{INTRODUCTION}

ICE-FORMATION phenomena are observed in many diverse processes, such as the freezing of water in pipes, the blockage of chemical process lines and the freezing of liquid metals in heat exchangers. Many theoretical and experimental studies have been performed for fluid flow with solidification in circular tubes and parallel plate channels and its effect upon laminar flow heat transfer [1-4]. On the other hand, there are only a few studies which deal with the prediction of the solidified crust in the presence of a turbulent flow. In the treatment of solidification in turbulent flow it was assumed that the increase in ice layer thickness in flow direction is very small, so that, as a first approximation, it does not affect the flow characteristics [5-7]. However, if the ice layer is not thin enough, there will be an interaction between the turbulent flow, the shape of the ice and the heat transfer at the ice-water interface. Under certain conditions these interactions result in an instability of the ice layer. This instability is caused by a strong laminarization of the turbulent flow due to an acceleration of the liquid as a consequence of converging ice layers in the entrance region of the cooled test section which results in a wavy ice structure. The morphology of ice structure in a circular pipe was investigated experimentally by Gilpin $[8,9]$ and by Hirata and Matsuzawa [10].

The ice-structure in a cooled parallel plate channel was first studied by Seki et al. [11], but they investigated only a small range of flow-rate Reynolds numbers and cooling parameters. Because of this fact, wavy ice layers with only one wave could be observed in their experıments. Weigand and Beer [12] extended the experimental study done by Seki et al. [11] and found that wavy ice layers with many waves occur also in a parallel plate channel for high values of the cooling ratio $\theta_{\mathrm{c}}$. In their experimental study, concerning the morphology of ice structure in a cooled two-dimensional channel, they observed that strong three-dimensional structures of the ice bands developed for very high values of the cooling parameter.

The purpose of this paper is to present the results of an experimental and theoretical study concerning steady-state ice formation inside a cooled paralle! plate channel. The focal point of the study is the presentation of a simple and quite flexible numerical model for predicting ice layers with one wave in the entrance region of the cooled channel.

\section{PHYSICAL MECHANISM}

Figure 1 shows the geometrical configuration and the coordinate system. The turbulent fluid flow enters the cooling section at $x=0$ with a fully developed velocity profile and with constant temperature $T_{0}$. In the chill region the wall temperature is maintained at a constant value $T_{\mathrm{w}}$, which is lower than the freezing temperature $T_{\mathrm{F}}$ of the fluid and a frozen layer is generated at the cooled walls.

Figure 2 shows a photograph of an ice layer with one wave for $R e_{4 h}=36000, P r=13$ and $\theta_{\mathrm{c}}=8$. The length of the channel is scaled in $\mathrm{cm}$ in Fig. 2 and the dimensionless quantities are defined as

$$
R e_{4 h}=\frac{\tilde{u}_{0} 4 h}{v_{\mathrm{L}}}, \quad \operatorname{Pr}=\frac{v_{\mathrm{L}}}{a_{\mathrm{L}}}, \quad \theta_{\mathrm{c}}=\frac{T_{\mathrm{F}}-T_{\mathrm{w}}}{T_{0}-T_{\mathrm{F}}}
$$

where $\bar{u}_{0}$ denotes the axial mean velocity at the 


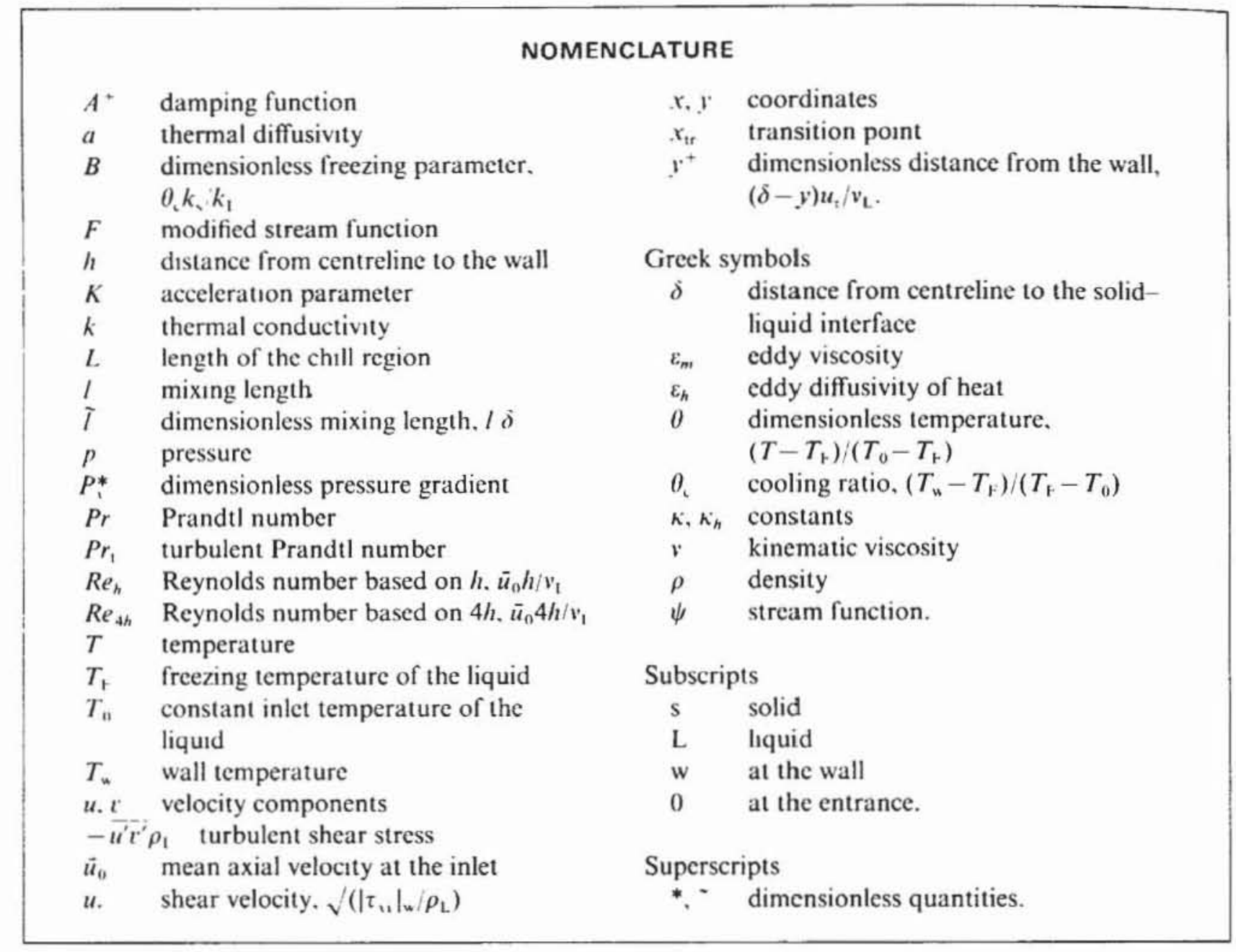

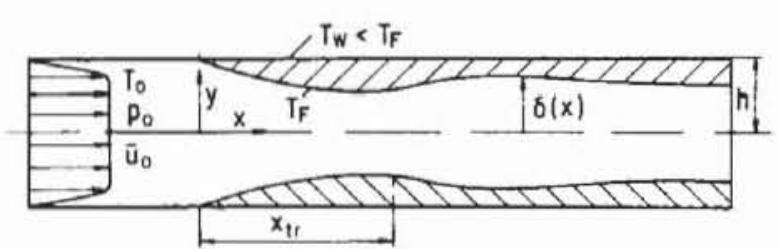

Fig. 1. Physical model and coordinate system.

entrance of the cooling section. The occurrence of one wave near the entrance of the test section can be explained as follows: during the growth of the ice layer the fluid undergoes a strong acceleration in the entrance section of the cooled channel. This acceleration tends to laminarize the flow. A criterion for the retransition of the flow was given by Moretti and Kays [13]. They found from experiments that a critical value of an acceleration parameter $K$, defined as

$$
K=\frac{v_{\mathrm{L}}}{\bar{u}^{2}} \frac{\mathrm{d} \bar{u}}{\mathrm{~d} x}=-\frac{1}{R e_{h}} \frac{\mathrm{d} \delta}{\mathrm{d} x}
$$

with $K_{\text {crtt }} \geqslant 2 \times 10^{-6}-3 \times 10^{-6}$, was sufficient to relaminarize an initially turbulent flow. For the ice layer shown in Fig. 2 values of $K>5 \times 10^{-6}$ occur near the entrance of the test section. Because of the flow laminarization, the laminar sublayer at the wall increases in thickness and the heat transfer from the flund to the solid crust diminishes. The thicker laminar sublayer is stabilized by the strong negative pressure gradient in axial direction. In a distance from the entrance the ice layers get nearly parallel and the acceleration of the fluid, which is proportional to $\mathrm{d} \delta / \mathrm{d} x$, tends to zero. Therefore. the stabilizing pressure gradi-

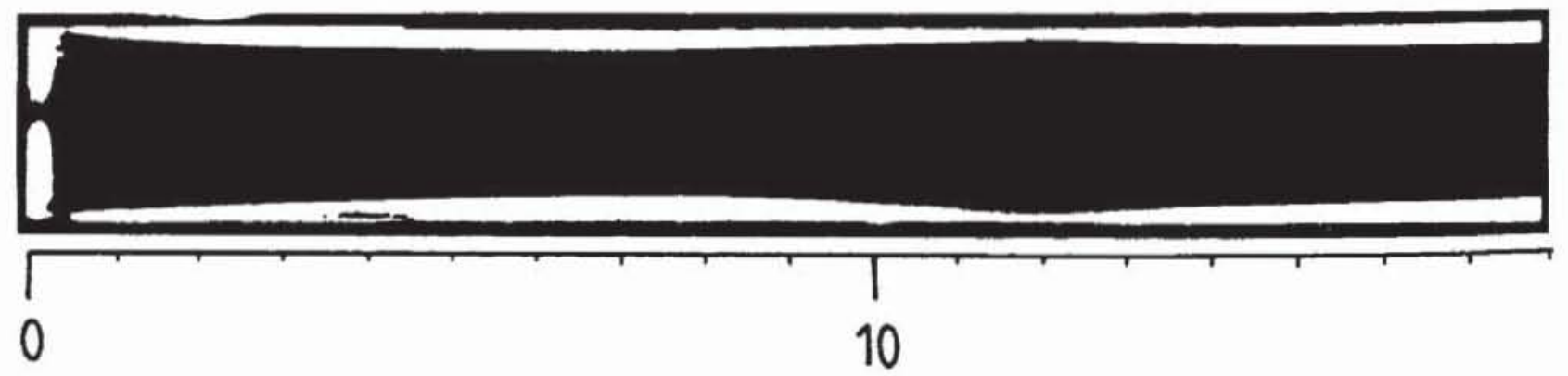

FIG 2. 'Smooth transition' ice layer for $R e^{\prime}=36000, \theta_{\mathrm{c}}=8$ and $\operatorname{Pr}=13$. 

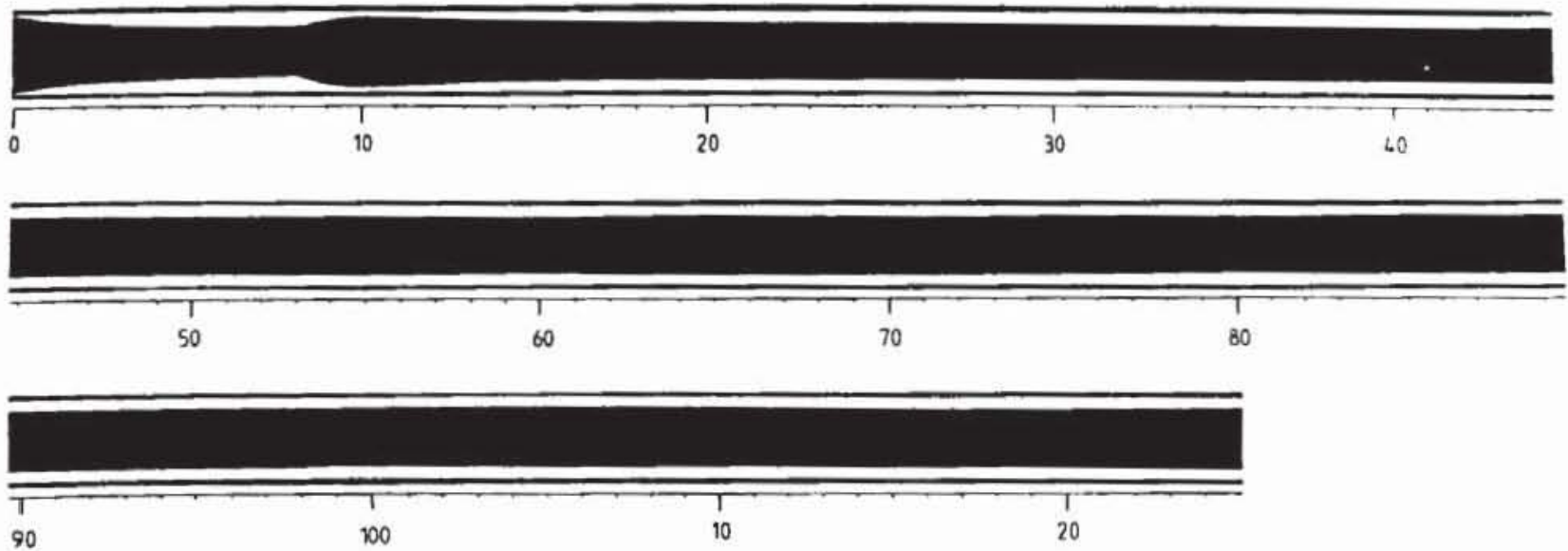

FIG. 3. 'Step transition' ice formation for $R e_{4 h}=39000.0_{\mathrm{c}}=104$ and $\operatorname{Pr}=13.2$

ent of the outer flow ceases and the fluid recedes to its originally turbulent state. The increase in heat transfer to the solid crust results in a decreasing ice layer thickness in this region. If the preceding lamınarization of the fluid was moderate only, the flow passage expands gradually in flow direction (Fig. 2). This type of ice formation is known in literature as 'smooth transition' ice formation. If the laminarization in the entrance region is more pronounced. the expansion of the flow passage leads to a flow separation in this region, which is due to the sharp increase of the cross-sectional area. This type of ice layer, which is depicted in Fig. 3. is designated as 'step transition' ice formation. After attaining mınımum thickness, the ice layer grows monotonously again with increasing axial coordinate (Fig. 3).

Following the study of Weigand and Beer [12]. wavy ice layers with one wave occur in a parallel plate channel for

$$
-0.41+\frac{R e_{4 h}}{7077} \leqslant 0, \leqslant 2.4+\frac{R e_{4 h}}{4421} . \quad R e_{4 h}>6000 .
$$

For a detailed description of the experimental apparatus, the operating procedure and the morphology of ice structure, the reader is referred to refs. [12. 22].

\section{ANALYSIS}

\subsection{Basic equations and assumptions}

By assuming an incompressible. Newtonian fiuid with constant fluid properties, the steady-state conservation equations for the fluid are cast into nondimensional form by using the following variables:

$$
\begin{aligned}
& x^{*}=\frac{x}{h}, \quad y^{*}=\frac{y}{h} \sqrt{ }\left(R e_{h}\right), \quad \delta^{*}=\frac{\delta}{h}, \quad \varepsilon_{m}^{*}=\frac{\varepsilon_{m}}{v_{\mathrm{L}}} \\
& u^{*}=\frac{u}{\tilde{u}_{0}}, \quad r^{*}=\frac{r}{\bar{u}_{0}} \sqrt{ }\left(R e_{h}\right), \quad p^{*}=\frac{p}{\rho_{\mathrm{L}} \bar{u}_{0}^{2}} . \quad \varepsilon_{h}^{*}=\frac{\varepsilon_{h}}{v_{\mathrm{L}}}
\end{aligned}
$$

$$
\theta=\frac{T-T_{1}}{T_{0}-T_{1}}, \quad P r_{1}=\frac{\varepsilon_{m}^{*}}{i i_{n}^{*}} .
$$

This results in

$$
\begin{aligned}
& \hat{c} u^{*}+\frac{\partial t^{*}}{\partial y^{*}}=0 \\
& u^{*} \frac{\hat{\partial} u^{*}}{\hat{c} x^{*}}+r^{*} \frac{\hat{c} u^{*}}{\hat{\imath} y^{*}}=-\frac{\hat{\partial} p^{*}}{\hat{c} x^{*}}+\frac{\hat{c}}{\hat{\partial} y^{*}}\left[\left(1+\varepsilon_{m}^{*}\right) \frac{\hat{c} u^{*}}{\hat{c} y^{*}}\right] \\
& 0=-\frac{\hat{c} p^{*}}{\hat{c} y^{*}} \\
& u^{*} \frac{\hat{c} \theta}{\hat{c} x^{*}}+r^{*} \frac{\hat{c} \theta}{\hat{\partial} y^{*}}=\frac{1}{\operatorname{Pr}} \dot{\hat{r} y^{*}}\left[\left(1+\frac{\operatorname{Pr}}{\operatorname{Pr}} \varepsilon_{t}^{*}\right) \frac{\hat{c} \theta}{\hat{c} y^{*}}\right]
\end{aligned}
$$

where the eddy viscosity $\varepsilon_{m}$, which appears in equations (6) and (8) is defined by

$$
-\rho_{1} \bar{u}^{\prime} \tau^{\prime}=\rho_{\mathrm{L}} \varepsilon_{m} \hat{\grave{\imath} u} .
$$

By deriving equations (5)-(8), the usual boundarylayer assumptions were made. which are a common treatment of the conservation equations for channel flows [14, 15]. The boundary conditions belonging to the conservation equations are

$$
\begin{aligned}
& x^{*}=0: u^{*}=\text { given. } \quad p^{*}=p_{0}^{*}, \quad \theta=1 \\
& y^{*}=0: \begin{array}{l}
\hat{\imath} u^{*} \\
\hat{i} y^{*}
\end{array}=\imath^{*}=0, \quad \frac{\hat{\theta} \theta}{\hat{\partial} y^{*}}=0 \\
& y^{*}=\delta^{*} \sqrt{ }\left(R e_{h}\right): u^{*}=r^{*}=0, \quad \theta=0 .
\end{aligned}
$$

In addition to the boundary conditions, the conservation of mass in integral form must be satisfied. By using the dimensionless quantities according to equation (4), the conservation of mass in integral form may be expressed in the following form: 


$$
\int_{0}^{s \cdot \sqrt{ }\left(R e_{h}\right)} u^{*} \mathrm{~d} y^{*}=\sqrt{ }\left(R e_{h}\right) .
$$

Furthermore, the energy equation for the solid region is required. By assuming constant properties in the solid phase and negligible axial conduction, the heat conduction equation for the solid region reduces to

$$
\frac{\partial^{2} T_{\mathrm{s}}}{\partial y^{2}}=0
$$

with the boundary conditions

$$
\begin{array}{ll}
y=\delta: & T_{\mathrm{s}}=T_{\mathrm{F}} \\
y=h: & T_{\mathrm{s}}=T_{\mathrm{w}} .
\end{array}
$$

Equations (5)-(11) and equations (12) and (13) are coupled by the interface energy equation, which adopts the following form for steady-state conditions :

$$
\left.k_{\mathrm{s}} \frac{\partial T_{\mathrm{s}}}{\partial y}\right|_{1=\delta}=\left.k_{\mathrm{L}} \frac{\partial T}{\partial y}\right|_{1=\delta} .
$$

\subsection{Temperature distribution in the solid region}

By using the boundary conditions according to equations (13), the temperature distribution in the solid phase is easily calculated from equation (12)

$$
T_{\mathrm{s}}=T_{\mathrm{w}} \frac{y-\delta}{h-\delta}+T_{\mathrm{F}} \frac{h-y}{h-\delta} ; \quad y \geqslant \delta .
$$

\subsection{Velocity and temperature distribution in the liquid}

After introducing a stream function, defined as

$$
u^{*}=-\frac{\partial \psi}{\partial y^{*}}, \quad v^{*}=\frac{\partial \psi}{\partial x^{*}}
$$

into equations (5)-(8) and by applying the coordinate transformation

$$
\tilde{y}=\sqrt{ }\left(R e_{h}\right)\left(1-\frac{y}{\delta}\right), \quad \tilde{x}=x^{*}
$$

to the conservation equations, the following set of partial differential equations can be obtained:

$$
\begin{aligned}
\frac{1}{\delta^{* 2}} \frac{\partial}{\partial \hat{y}}\left[\left(1+\varepsilon_{m}^{*}\right) \frac{\partial^{2} F}{\partial \tilde{y}^{2}}\right] & =\frac{\partial p^{*}}{\partial \tilde{x}}+\frac{\partial F}{\partial \tilde{y}} \frac{\partial^{2} F}{\partial \tilde{x} \hat{y} \tilde{y}} \\
& -\frac{\partial F}{\partial \tilde{x}} \frac{\partial^{2} F}{\partial \tilde{y}^{2}}-\frac{1}{\delta^{*}} \frac{\mathrm{d} \delta^{*}}{\mathrm{~d} \tilde{x}} F \frac{\partial^{2} F}{\partial \tilde{y}^{2}} \\
0 & =\frac{\partial p^{*}}{\partial \tilde{y}}
\end{aligned}
$$

$$
\begin{aligned}
\frac{1}{\delta^{* 2}} \frac{\partial}{\partial \tilde{y}}[ & \left.\left(1+\frac{\operatorname{Pr}}{\operatorname{Pr}_{1}} \varepsilon_{m}^{*}\right) \frac{\partial \theta}{\partial \tilde{y}}\right] \\
& =\operatorname{Pr}\left[\frac{\partial F}{\partial \tilde{y}} \frac{\partial \theta}{\partial \tilde{x}}-\frac{\partial F}{\partial \tilde{x}} \frac{\partial \theta}{\partial \tilde{y}}-\frac{1}{\delta^{*}} \frac{\mathrm{d} \delta^{*}}{\mathrm{~d} \tilde{x}} F \frac{\partial \theta}{\partial \tilde{y}}\right] .
\end{aligned}
$$

In equations (18)-(20) the modified stream function

$$
F=\frac{\psi}{\delta^{*}}
$$

was used. The boundary conditions belonging to equations (18)-(20) are given by

$$
\begin{aligned}
& \tilde{x}=0: \frac{\partial F}{\partial \tilde{y}}=\text { given, } \quad p^{*}=p_{0}^{*}, \quad \theta=1 \\
& \tilde{y}=0: \frac{\partial F}{\partial \tilde{y}}=0, \quad F=0, \quad 0=0 \\
& \tilde{y}=\sqrt{ }\left(R e_{h}\right): \frac{\partial^{2} F}{\partial \tilde{y}^{2}}=0, \quad F=\sqrt{ }\left(R e_{h}\right) / \delta^{*}, \quad \frac{\partial \theta}{\partial \tilde{y}}=0 .
\end{aligned}
$$

The coordinate transformation according to equation (17) is very useful in order to solve the conservation equations, because the duct with variable distance between the wall and the centreline is transformed into a duct with constant height.

The conservation of mass in integral form, given by equation (11), results in an additional boundary condition for the modified stream function $F$ at $\tilde{y}=$ $\sqrt{ }\left(R e_{h}\right)$, as it can be seen in equation (22).

The energy balance at the solid-liquid interface equation (14), can be rewritten after inserting the temperature distribution in the solid phase

$$
\left.\frac{\partial \theta}{\partial \tilde{y}}\right|_{\dot{i}=0}=\frac{B}{\sqrt{ }\left(R e_{h}\right)} \frac{\delta^{*}}{1-\delta^{*}} .
$$

The dimensionless freezing parameter $B$, which was used in equation (23), is defined as

$$
B=\frac{k_{\mathrm{s}}}{k_{\mathrm{L}}} \frac{T_{\mathrm{F}}-T_{\mathrm{w}}}{T_{0}-T_{\mathrm{F}}}=\frac{k_{\mathrm{s}}}{k_{\mathrm{L}}} \theta_{\mathrm{c}}
$$

where $k_{\mathrm{s}} / k_{\mathrm{L}}$ can be taken approximately as 4 for the temperature range of $T_{w}$ and $T_{0}$ under investigation.

3.3.1. Turbulence modelling. By predicting the axial distribution of the ice layer thickness one of the major problems arises due to the modelling of the eddy viscosity $\varepsilon_{m}^{*}$ in equations (18) and (20). For the fluid flow and heat transfer in a parallel plate channel containing a wavy ice layer with one wave, there are essentially three distinct regions. For small values of the axial coordinate $\left(x<x_{t r}\right.$, region 1$)$ the flow undergoes a strong acceleration and flow laminarization can be observed (Fig. 1). The flow behaviour in region III $\left(x \gg x_{\mathrm{r}}\right)$ is approximately the same as that in a parallel plate channel, because $\mathrm{d} \delta / \mathrm{d} x$ changes only slightly in this area. In region II both models have to be matched. Therefore, turbulence models will be formulated independently for each of the mentioned areas.

Highly accelerated flow (region I). Many papers can be found in literature which deal with the laminarization of an initially fully turbulent flow in a highly accelerated boundary layer flow. A summary is given in ref. [16].

Measurements of the Reynolds shear stress $-\rho_{\mathrm{L}} \overline{u v}$ in a highly accelerated flow show that the turbulent 
shear stress is approximately constant along a streamline [16]. This experimental observation is in good agreement with the theory developed by Deissler [17], in which he examined the two-point equations for the turbulent stresses. After transforming the equations into von Mises coordinates $(x, \psi)$ and integrating the resulting equations, he found out that

$$
\left.\overline{u^{\prime} v^{\prime}}(\psi) \approx \overline{u^{\prime} v^{\prime}}(\psi)\right)_{x=0}
$$

for $\psi=$ const. and for sufficient high values of the acceleration parameter $K$. This shows that the Reynolds shear stress can be considered to be frozen at its initial values as it is convected along the streamlines (for more detailed information the reader is referred to ref. [17]).

It is interesting to note that the turbulence model according to Deissler [17] involves no empirical functions or constants. The description of the initial distribution of the Reynolds shear stress for $x=0$ suffices to calculate the axial development of the velocity profiles in the highly accelerated boundary layer flow. Because the flow enters the cooled test section with a fully developed, turbulent velocity profile, the Reynolds shear stress, $\left(-\rho_{\mathrm{L}} \overline{u^{\prime} v^{\prime}}\right)_{r-0}$, can be calculated easily by applying the mixing length model originally developed by Prandtl $[14,15]$.

Mixing length model (region III). In region 1II, the turbulent shear stress, $-\rho_{\mathrm{L}} \bar{u}^{\prime} v^{\prime}$, can be modelled with the help of a modified mixing length theory developed by Moretti and Kays [13]. The eddy viscosity is given in dimensionless quantıties by

$$
\left(\varepsilon_{m}^{*}\right)_{\mathrm{III}}=\delta^{*}\left(R e_{h}\right)^{3}{ }^{2} \tilde{l}^{2}\left|\frac{\hat{c}^{2} F}{\partial \tilde{y}^{2}}\right|
$$

where the well-known mixing length formula of Nikuradse with the damping factor, according to van Driest

$$
\begin{aligned}
\tilde{I}=[0.14 & -0.08\left(1-\frac{\tilde{y}}{\sqrt{ }(\operatorname{Re})_{h}}\right)^{2} \\
& \left.\quad-0.06\left(1-\frac{\tilde{y}}{\sqrt{ }\left(R e_{h}\right)}\right)^{4}\right]\left(1-\mathrm{e}^{-y^{+} A^{+}}\right)
\end{aligned}
$$

was used in equation (26). The damping function $A^{+}$ is evidently determined by viscous stability considerations. A favorable pressure gradient (acceleration) results in an increasing thickness, while an adverse pressure gradient has the opposite effect. Moffat and Kays [18] attributed the failure in modelling $A^{+}$correctly as the main reason for the bad repute of mixing length models in the past.

Following the work of Moffat and Kays [18], $A^{+}$ is modelled as

$$
\frac{\mathrm{d} A^{+}}{\mathrm{d} \tilde{x}}=\frac{R e_{\mathrm{\tau}}}{C}\left(A_{\mathrm{e}}^{+}-A^{+}\right)
$$

with the function $A_{\mathrm{c}}^{+}$

$$
A_{\mathrm{c}}^{+}=\frac{25}{1+a P^{*}} \text {. }
$$

The dimensionless pressure gradient $P_{i}^{*}$ is defined by

$$
P_{r}^{*}=\frac{R e_{h}^{2}}{\operatorname{Re}_{\mathrm{t}}^{3}} \frac{1}{\delta^{* 3}} \frac{\mathrm{d} \delta^{*}}{\mathrm{~d} \tilde{x}}
$$

with the Reynolds number $R e_{\tau}$ based on the friction velocity

$$
R e_{\tau}=\frac{u_{\mathrm{\tau}} h}{v_{\mathrm{L}}}=\sqrt{\left(\frac{\left|\tau_{\mathrm{v}}\right|_{\mathrm{u}}}{\rho_{\mathrm{L}}}\right) \frac{h}{v_{\mathrm{L}}} .}
$$

The constants $a$ and $C$, appearing in equation (28), have the values

$$
\begin{array}{lll}
P_{\mathrm{r}}^{*}<0: & a=37.2, & C=2000 \\
P_{\mathrm{r}}^{*}>0: & a=20.59, & C=1000 .
\end{array}
$$

The function $A_{\mathrm{c}}^{+}$represents the values which $A^{+}$ adopts under essentially equilibrium conditions. The influence of the pressure gradient on $A_{\mathrm{e}}^{+}$, according to equation (29), was modelled from experimental data by Moffat and Kays [18].

In any case, under non-equilibrium conditions, it has been observed that the sublayer does not change instantaneously to its new equilibrium thickness. Therefore, the rate equation (28) was used to incorporate relaxation of the turbulent boundary layer.

Region II. If the turbulence model according to Deissler [17] would be valid throughout the whole axial region, a monotonously decreasing function for $\delta^{*}$ could be obtained (Fig. 4). Figure 4 shows that the acceleration parameter $K$, defined by equation (2), decreases sharply with growing values of $\tilde{x}$. For $\tilde{x} \geqslant 8$ the parameter $K$ falls short of $2 \times 10^{-6}$, which is the critical value of $K$ for which laminarization phenomena can be observed in the flow. On the other hand experiments reveal that the ice layer thickness decreases for $\tilde{x} \geqslant \tilde{x}_{\mathrm{tr}}$, which can be attributed to the again increasing heat transfer from the fluid to the solid-liquid interface. The retransition of the laminarized fluid flow to the turbulent state is caused by instabilities occurring in the laminar sublayer for $\tilde{x} \geqslant \tilde{x}_{\text {cru }}$, because the stabilizing effect of the strongly negative pressure gradient is no longer present. This

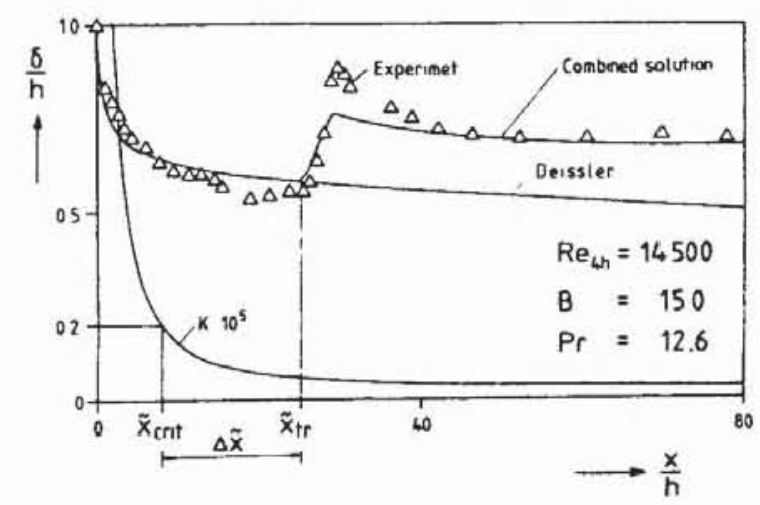

Fig. 4. Wavy ice layer for $R e_{4 h}=14500, B=15$ and $\operatorname{Pr}=12.6$. 
retransition to a fully turbulent flow behaviour is quite similar to the transition phenomena of a laminar fluid flow to the turbulent state (for details see ref. [16]). Therefore, the eddy viscosity of region II can be described by the following expression :

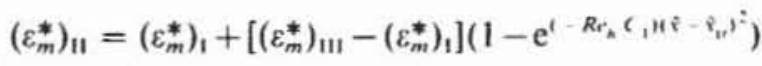

$$
\begin{aligned}
& C_{1}=3 \times 10^{4} . \quad \tilde{x}>\tilde{x}_{t r}
\end{aligned}
$$

in analogy to the transition of a laminar flow to a fully turbulent flow [15]. Equation (33) adapts the eddy viscosity $\left(\varepsilon_{m}^{*}\right)$, for region I, given by equations (25) and (9). with that for region III, given by equation (26).

In equation (33) $\tilde{x}_{t r}$ is yet unknown. It might be possible to express $\tilde{x}_{\mathrm{tr}}$ as a function of $R e_{h}$ and $B$, as it was done by Seki et al. [11], using their experimental data. This expression could be used directly in equation (33). However, from a physical point of view it secms more ingenıous to model the axial distance as $\Delta \tilde{x}=\tilde{x}_{1 r}-\tilde{x}_{\text {crt }}$, because this distance describes the region in which instabilities develop in the laminar sublayer. From measurements we obtained the following expressions for $\Delta \tilde{x}$ :

$$
\Delta \tilde{x}=1.4 \times 10^{6} R e_{h}^{-32} B^{-12} .
$$

In order to relate the eddy diffusivity of heat $\varepsilon_{n}^{*}$. which appears in the energy equation, to the eddy viscosity $\varepsilon_{m}^{*}$, a formulation for the turbulent Prandt number is required. In this study the turbulent Prandtl number concept according to Cebeci [14] was used

$$
P r_{1}=\frac{\kappa}{\kappa_{h}} \frac{1-\exp \left(-y^{+} / A_{0}^{+}\right)}{1-\exp \left(-y^{+} / B^{+}\right)}
$$

with the quantities

$$
\begin{aligned}
\kappa & =0.4 . \quad \kappa_{h}=0.44, \quad A_{0}^{+}=25 \\
B^{+} & =\frac{1}{\sqrt{(P r})} \sum_{i=1}^{5} C_{1}(\log P r)^{r-1} \\
C_{1} & =34.96, \quad C_{2}=28.79, \quad C_{3}=33.95, \\
C_{4} & =6.33 . \quad C_{5}=-1.186 .
\end{aligned}
$$

\section{NUMERICAL METHOD AND CALCULATION PROCEDURE}

The calculation procedure can be split up in several parts. First, an ice layer is calculated under the assumption that the turbulence model according to Deissler [17] is valid throughout the whole chill region. For this, an intial distribution of $\delta^{*}(\tilde{x})$ is needed. We used

$$
\begin{gathered}
\delta^{*}(\tilde{\xi})=1\left(1+B_{\vee}(\pi \tilde{\xi})\right) \\
\dot{x}=\frac{x}{h}=\frac{2 \operatorname{Re}_{h} \operatorname{Pr}}{\pi B^{2}}\left[B_{\checkmark}(\pi \xi)\right. \\
\left.\quad-\ln \left(1+B_{\checkmark}(\pi \xi)\right)\right] . \quad 0 \leqslant \xi \leqslant 0.2
\end{gathered}
$$

as an assumption for the inttial ice layer thickness.
The variation of $\delta^{*}$ given by equations (37) and (38), can be obtained as an approximation of $\delta^{*}$ proposed by Lee and Zerkle [2] for a slug flow velocity profile.

With the assumed distribution of $\delta^{*}(\tilde{x})$ and the turbulence model according to Deissler, equation (25), the velocity and temperature distribution in the liquid can be calculated from equations (18) to (20) in conjunction with the boundary conditions, equation (22).

Solutions of the conservation equations were obtained with the help of an implicit finite-difference method, which is known in literature as the Kellerbox method [14. 15]. This method has several very desirable features that make it appropriate for the solution of all parabolic partial differential equations; one of which is that it allows highly non-uniform $\tilde{x}$ and $\tilde{y}$ spacings by second-order accuracy. Because the box scheme is a common method for solving parabolic differential equations, only a brief outline is provided here [14]. First of all the parabolic differential equation of order $n$ is reduced to a system of $n$ first-order equations. These equations were approximated by difference equations using central differences at each nodal point. The ensuing algebraic equations were linearized. This results in a linear system of equations, which show a block tridiagonal structure and can be solved easily $[14,15]$.

After solving the conservation equations. a new distribution of $\delta^{*}(\tilde{x})$ is calculated by inserting the yet known temperature gradient at the solid-liquid interface into equation (23). With the new distribution of $\delta^{*}(\tilde{x})$, the conservation equations (18)-(20) are solved again, and so on. The iteration procedure described above was repeated until the deviation between two successive ice layers was within

$$
\Delta \delta^{*}=\left|\delta^{*(1)}-\delta^{*(1-1)}\right|<0.01
$$

at every axial position. The iteration process converges rapidly. Only three to four iterations were necessary to get $\delta^{*}(\tilde{x})$ within the error bound given above.

After calculating the variation of $\delta^{*}(\tilde{x})$, which is a monotonously decreasing function of $\tilde{x}$ (see Fig. 4), the variation of the acceleration parameter $K$ can be predicted from equation (2). For $K=2 \times 10^{-6}$ the axial position of $\hat{x}_{\mathrm{cnt}}$ is determıned (Fig. 4). Finally, the transition point $\tilde{x}_{t r}$ is found from equation (34). With the knowledge of $\tilde{x}_{t r}$ the distribution of the ice layer thıckness in regions II and III can now be calculated with the help of the turbulence models described in the preceding chapter. The iteration procedure for obtaining the ice layer thickness in regions II and III is actually the same as that described above.

The calculations were performed with the help of a strongly non-uniform grid in $\hat{x}$ and $\tilde{y}$ directions. Numerical runs showed that approximately 80 grid points in $\tilde{y}$ direction and nearly 200 points in axial direction guaranteed sufficient accuracy. The length of the chill region was $L / h=80$.

For higher values of the cooling parameter $B$, the 
cross-sectional area increases sharply for $\hat{x}>\hat{x}_{\mathrm{tr}}$ and flow separation may occur in this region. Figure 3 shows a 'step transition' Ice formation for $R e_{\text {sh }}=39000 . P r=13.2$ and $\theta_{\mathrm{t}}=10.4$. For this case, the FLARE approximation of Reyhner and FlüggeLotz [19] was applied to represent small regions of separated flow. The use of boundary layer equations to represent separated flows presumes that the pressure gradient in normal direction and longitudinal diffusion are negligible in the regions of recirculation. Also, it must be assumed that the regions of recirculation remain small as compared to the characteristic length of the problem. In the present case, the error introduced by applying the FLARE approximation in the small recirculation regions can be neglected with good accuracy (for more detailed information, see Kwon et al. [20]).

\section{RESULTS AND DISCUSSION}

Figure 5 shows a 'smooth transition' ice formation for $R e_{4 h}=20000, \operatorname{Pr}=13$ and $B=16$. For stcady state conditions the experimental results are compared with the numerical analysis. It can be seen that the ice layer thickness increases sharply for low values of the axial coordinate, whereas it reaches a nearly constant thickness for larger values of $\tilde{x}$. The axial position of the transition point is found to be approximately $\tilde{x}_{\mathrm{tr}}=16$. For $\tilde{x}>\tilde{x}_{\mathrm{tr}}$ the ice layer first decreases in thickness. After attaining minimum thickness, the ice layer grows gradually again with increasing axial coordinate and approaches a nearly constant thickness for $\tilde{x} \geqslant 40$. Figure 5 elucidates that the theoretical results are in good agreement with our experimental data.

In Fig. 6 the development of the axial velocity profile is shown for the ice layer of Fig. 5. The axial velocity is scaled with the axial mean velocity at the entrance of the test section. It can be observed that the maximum velocity at the centreline of the channel increases for $\tilde{x}<\tilde{x}_{t r}$, which is due to the acceleration of the fluid caused by the converging ice layers. In the diverging channel region, the maximum velocity decreases but no flow scparation occurs. For larger

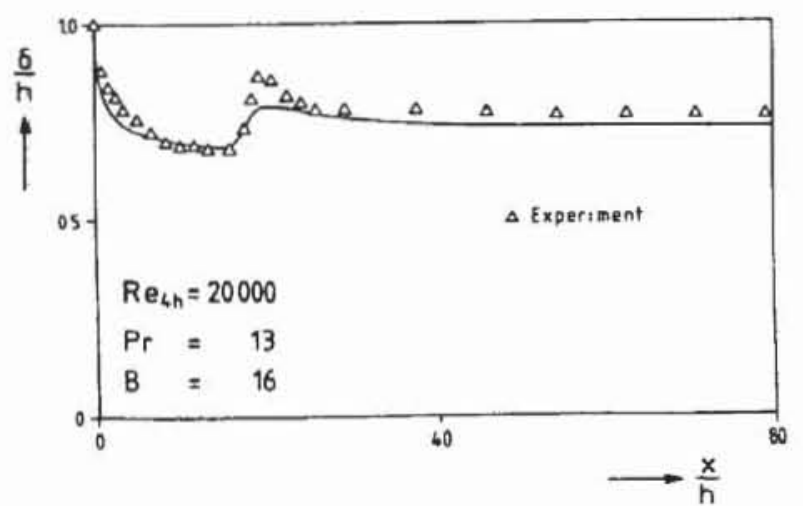

Fig. 5 'Smooth transition' ice formation for $R e_{4 h}=20000$. $B=16$ and $\operatorname{Pr}=13$.

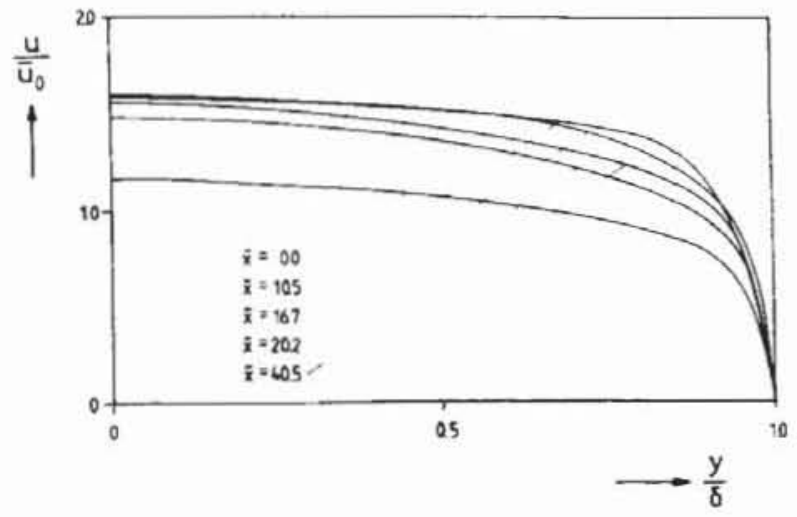

Fig 6 Axial velocity distribution as a function of $y \delta$ for various $x h$

values of the axtal coordinate, the centreline velocity increases slowly with growing values of $\dot{x}$.

Figure 7 shows the distribution of the Reynolds shear stress at several selected streamlines $(\psi=$ const. $)$ for the ice layer plotted in Fig. 5 . It can be seen that $u^{\prime} v^{\prime}$ remains constant along a streamline for $\hat{x}<\tilde{x}_{t r}$, which was presumed in equation (25). In the divergent channel region the Reynolds shear stress is strongly enhanced. For larger values of the axial coordinate $u^{\prime} t^{\prime}$ takes nearly constant values as it is convected along a streamline.

Figure 8 shows the influence of an increasing wall cooling parameter $B$ on the shape of the ice layer for various Reynolds numbers. It is obvious that an increasing wall cooling parameter $B=k_{\mathrm{s}} / k_{\mathrm{L}} \theta_{\mathrm{c}}$ results in a thicker ice layer for a given value of the Reynolds number. For $R e_{4 h}=20000$ it can be observed that a smooth ice layer develops in the channel for $B=8$. Increasing $B$ leads to a 'smooth transition' ice formation $(B=12.5)$. If $B$ is further increased $(B=22.5)$, the flow passage expands very sharply for $\tilde{x}>\tilde{x}_{\mathrm{tr}}$ and a 'step transition' ice structure is obtained. It is interesting to note that the distance $\tilde{x}_{\mathrm{tr}}$ between the entrance of the chill region and the point for which the ice layer reaches its maximum thickness seems to be only a function of the Reynolds number. This fact is elucidated by Fig. 8. Another interesting detail is the observation that the minimum thickness of the ice layers also seems to depend only on the Reynolds number and not on the cooling parameter $B$.

Figure 9 elucidates the effect of a variation in Reyn-

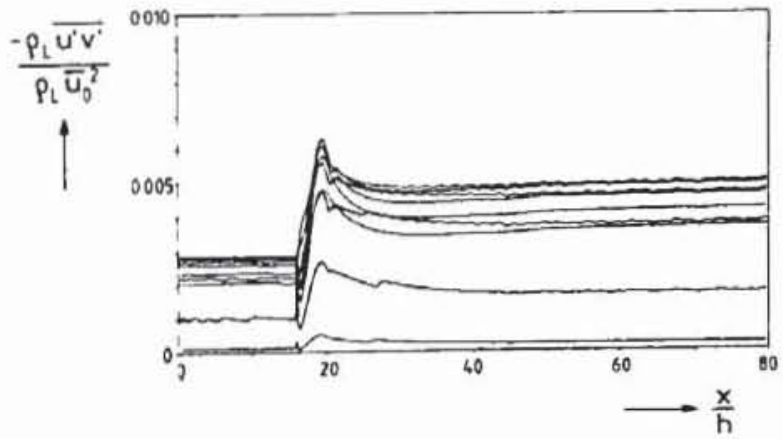

Fig. 7. Axial distribution of the Reynolds shear stress for several streamlines. 

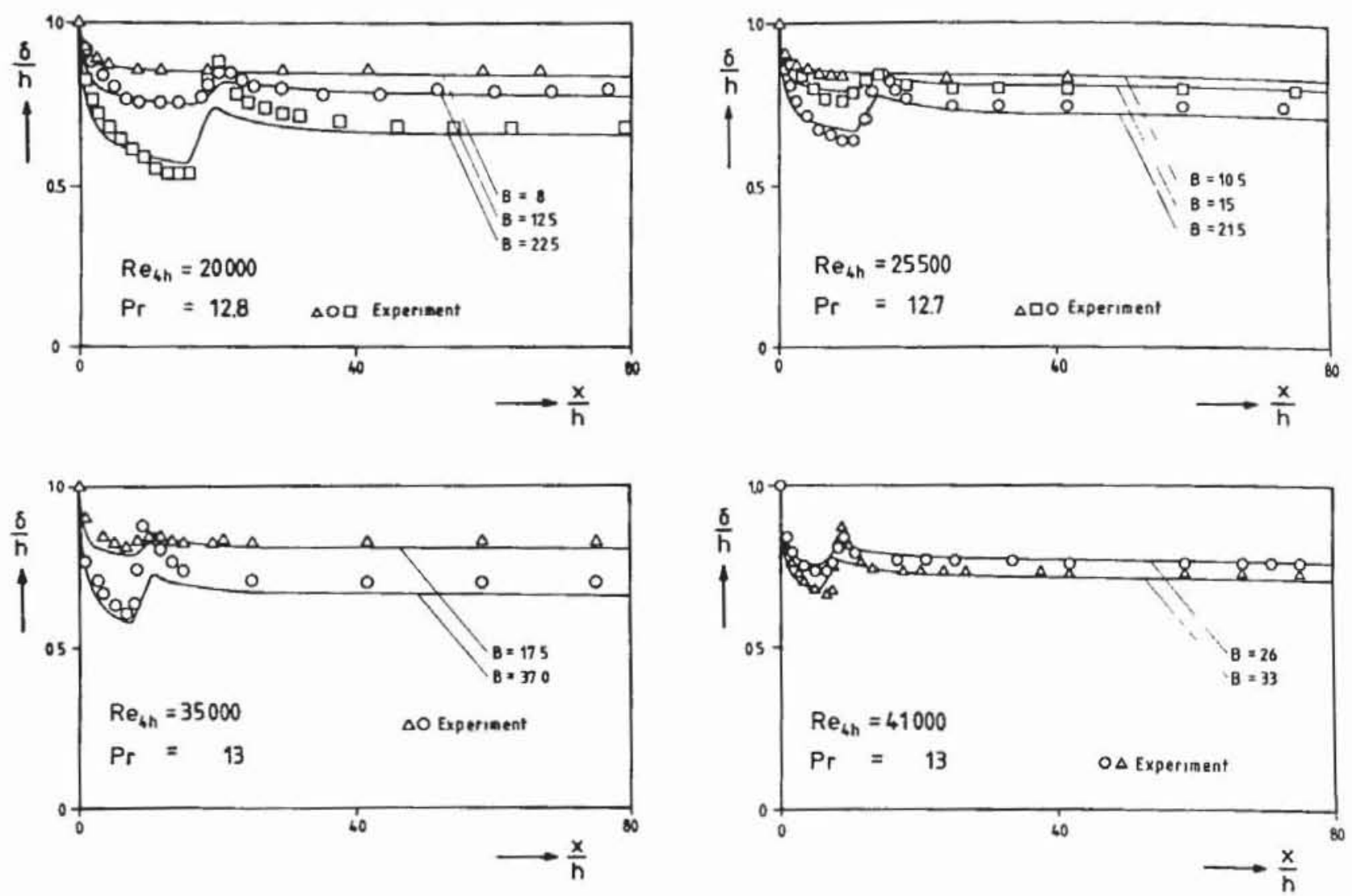

FIG. 8. Influence of the coolıng parameter $B$ on the shape of the ice layers.

olds number for $B=$ const. and a specified Prandtl number. It is obvious that an increasing Reynolds number results in a decreasing ice layer thickness. This can be attributed to the intensified heat transfer from the flowing liquid to the ice layer with growing Reynolds numbers. Increasing the Reynolds number for constant values of $\operatorname{Pr}$ and $B$, therefore, tends to stabilize the ice layer and suppresses the development of wavy ice layers. Further, it can be seen that an increasing value of the Reynolds number results in a decreasing value of $\tilde{x}_{t r}$.

In the previous plots the scaling of the $\tilde{y}$ axis, as compared to the $\tilde{x}$ axis, was extremely stretched. To give a more realistic impression of the geometric relations, Fig. 10 shows two ice layers with the $\bar{y}$ axis less distorted.

As can be seen from Figs. 4 to 10 , the agreement between theory and experiment is quite good, except in the region where the ice layer reaches its minimum thickness. At this location the deviations between the numerical solution and the measurement are more pronounced. This fact can be attributed to the following reasons :

- If flow separation occurs for larger values of $B$, three-dimensional effects occur in the expansion region of the flow cross-section.

- For larger values of the diffuser angle, the axial
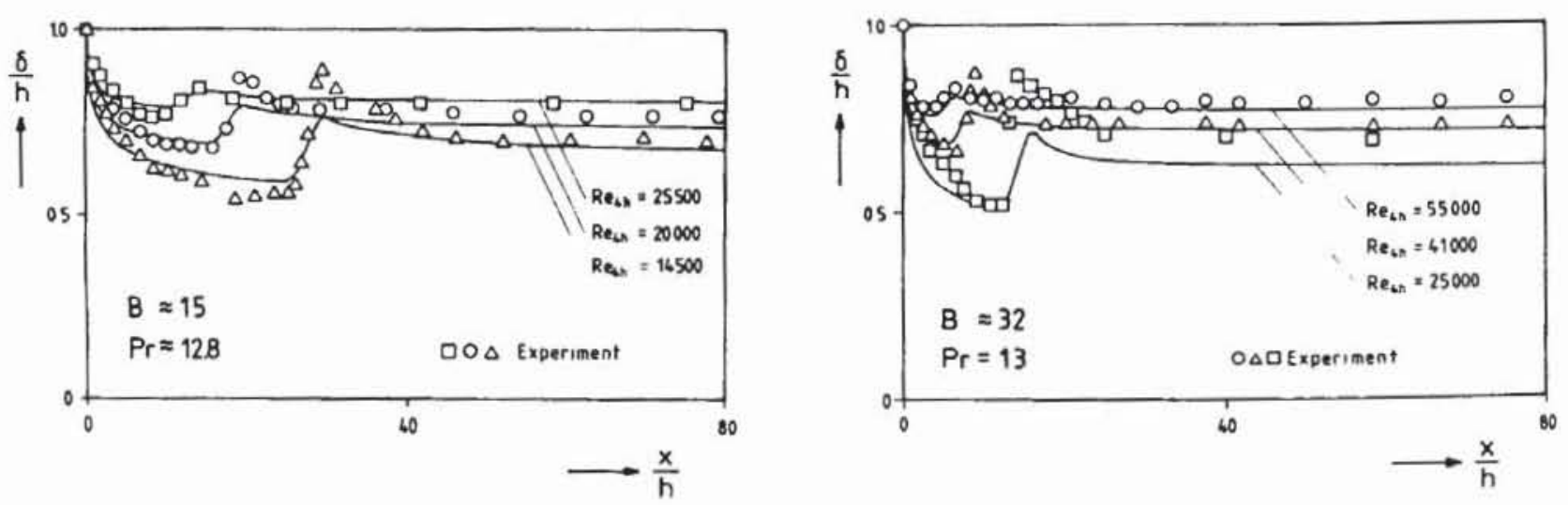

FIG. 9. Effect of Reynolds number on the axial distribution of $\delta^{*}$. 

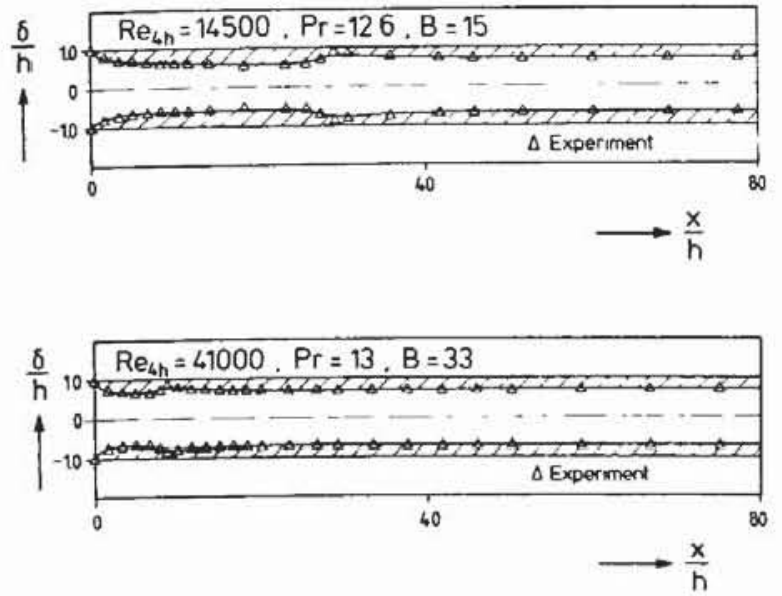

FIG. 10. Ice layer thickness as a function of $x / h$.

velocity profile can be asymmetric to the centreline of the channel, as it was observed by Nikuradse [21].

- The simple modified mixing length theory might not be able to model correctly the eddy viscosity in the recirculation region.

Nevertheless, the presented numerical method is able to predict realistic variations of the ice layer thickness for a wide range of Reynolds numbers and cooling parameters $B$.

\section{CONCLUSIONS}

According to the expenmental and theoretical results of the present investrgation concerning the freezing of water flow between parallel plates with the occurrence of laminarization effects, the following major conclusions may be drawn:

(1) A strong coupling exists between the nature of the flow and the shape of the solid-liquid interface. The development of wavy ice layers can be attributed to the flow laminarization in the entrance region of the parallel plate channel.

(2) Increasing the Reynolds number for given values of $B$ and $\operatorname{Pr}$ tends to stabilize the ice layer.

(3) The transition point $\tilde{x}_{t r}$ can be considered to depend solely on $R_{4 h}$.

(4) A simple method is presented for calculating the axial distribution of wavy ice layers with one wave for steady-state conditions.

Acknowledgement-The support of this work by the Deutsche Forschungsgememschaft is greatly acknowledged.

\section{REFERENCES}

1. R D. Zerkle and J. E. Sunderland, The eflect of liquid solidification in a tube upon laminar-flow heat transfer and pressure drop, J. Heat Transfer 90, 183-190 (1968).
2. D. G Lee and R D Zerkle. The effect of liquid solidification in a parallel plate channel upon laminar-flow heat transfer and pressure drop. $J$ Heat Transter 91 , 583-585 (1969)

3. Y. Kıkuchi, Y. Shigemasa, A. Oe and T. Ogata. Steadystate freezing of liquids in lamınar flow between two parallel plates, J. Nucl. Scl. Technol 23, 4355 (1986)

4. B. Weigand and H. Beer, Heat transfer and solidification of a laminar liquid flow in a cooled parallel plate channel: the stationary case, Warme- und Stoffubertragung 26, 233-240 (1991).

5. C. Cho and M. Özısik, Transient freezıng of lıquids in turbulent flow inside tubes. J. Heat Transfer 101, 456468 (1979).

6 A Shibanı and M Özisık, A solutıon of freezing of liquids of low Prandtl number in turbulent flow between parallel plates, J. Heat Transter 99, 20-24 (1977).

7. B. Weigand and H. Beer, Freezing in turbulent flow inside tubes and channels, Wàrme- und Stoffübertragung 28, 57-64 (1993)

8. R. R. Gilpın, The morphology of ice structure in a pıpe at or near transition Reynolds numbers. Heat TransferSan Diego 1979, A.I Ch.E Symp. Ser. 189, 89-94 (1979)

9. R. R. Gilpın. Ice formation in a pipe containing flows in the transition and turbulent regimes, $J$ Heat Transfer 103, 363-368 (1981).

10. T. Hirata and H. Matsuzawa, A study of ice-formation phenomena on freezing of flowing water in a pipe. $J$. Heat Transfer 109, 965-970 (1987)

11. N Sekı, S. Fukusako and G. W. Younan, Ice formation phenomena for water flow between two cooled parallel plates, J. Heat Transfer 106, 498-505 (1984).

12. B. Weigand and H. Beer, The morphology of ice structure in a parallel plate channel, Proc. 3rd Int. Simp. Cold Regions Heat Transfer, Fairbanks, Alaska, U.S.A., pp. 167-176 (1991).

13. P. M Morettı and W. M. Kays, Heat transfer to a turbulent boundary layer with varying free-stream velocity and varying surface temperature - an experimental study, Int. J. Heat Mass Transfer 8, 1187-1202 (1965).

14. T. Cebeci and P. Bradshaw, Physical and Computational Aspects of Contectute Heat Transfer. Springer, New York (1984).

15 T. Cebect and K. C. Chang. A general method for calculatung momentum and heat transfer in laminar and turbulent duct flows. Numer. Heat Transfer 1, 39-68 (1978).

16. R. Narasımha and K. R. Sreenıvasan, Relamınarization of fluid flows, Adt Appl. Mech. 19, 221-309 (1979)

17 R. G. Deissler, Evolution of a moderately short turbulent boundary layer in a severe pressure gradient, $J$. Flutd Mech. 64, 763-774 (1974).

18. R J Moffat and W M. Kays, A review of turbulentboundary-layer heat transfer research at Stanford, 19581983, Adr. Heat Transfer 16, 242-366 (1984).

19. T. A. Reyhner and I Flügge-Lotz, The interaction of a shock wave with a lamınar boundary layer, Int. J. NonLinear Mech. 3, 173-199 (1968).

20 O K. Kwon, R. H. Pletcher and J. P. Lewis, Prediction of sudden expansion flows using the boundary-layer equations, J. Fluid Engng 106, 285-291 (1984).

21. J. Nikuradse, Untersuchungen über die Strömung des Wassers in konvergenten und divergenten Kanälen, Forsch. Arb. Ing.-Wes. 289, 1-49 (1929).

22. B. Weigand, Erstarrungsvorgänge einer strömenden Flüssıgkeit in eınem ebenen, geraden Kanal, Doctoral Thesis, TH Darmstadt (1991). 